\title{
Selection properties of the split interval and the Continuum Hypothesis
}

\author{
Taras Banakh ${ }^{1,2}$
}

Received: 17 November 2019 / Accepted: 13 May 2020 / Published online: 3 June 2020

(c) The Author(s) 2020

\section{Abstract}

We prove that every usco multimap $\Phi: X \rightarrow Y$ from a metrizable separable space $X$ to a GO-space $Y$ has an $F_{\sigma}$-measurable selection. On the other hand, for the split interval $\ddot{\mathbb{I}}$ and the projection $P: \ddot{\mathbb{I}}^{2} \rightarrow \mathbb{I}^{2}$ of its square onto the unit square $\mathbb{I}^{2}$, the usco multimap $P^{-1}: \mathbb{I}^{2} \multimap \ddot{\mathbb{I}}^{2}$ has a Borel $\left(F_{\sigma}\right.$-measurable) selection if and only if the Continuum Hypothesis holds. This $\mathrm{CH}$-example shows that know results on Borel selections of usco maps into fragmentable compact spaces cannot be extended to a wider class of compact spaces.

Keywords Continuum hypothesis · Split interval $\cdot$ Measurable selection · Borel selection · usco multimap · Fragmentable compact · Rosenthal compact · GO-space

Mathematics Subject Classification 54C65 $\cdot$ 54F05 $\cdot$ 54H05 $\cdot 03$ E50

\section{Introduction}

By a multimap $\Phi: X \multimap Y$ between topological spaces $X, Y$ we understand any subset $\Phi \subseteq X \times Y$, which can be thought as a function assigning to every point $x \in X$ the subset $\Phi(x):=\{y \in Y:\langle x, y\rangle \in \Phi\}$ of $Y$. For a subset $A \subseteq X$ we put $\Phi[A]=\bigcup_{x \in A} \Phi(x)$. Each function $f: X \rightarrow Y$ can be thought as a single-valued multimap $\{\langle x, f(x)\rangle: x \in X\} \subseteq X \times Y$.

For a multimap $\Phi: X \multimap Y$, its inverse multimap $\Phi^{-1}: Y \multimap X$ is defined by $\Phi^{-1}:=\{\langle y, x\rangle:\langle x, y\rangle \in \Phi\}$.

A multimap $\Phi: X \multimap Y$ is called

- lower semicontinuous if for any open set $U \subseteq Y$ the set $\Phi^{-1}[U]$ is open in $X$;

- upper semicontinuous if for any closed set $F \subseteq Y$ the set $\Phi^{-1}[F]$ is closed in $X$;

\footnotetext{
$凶$ Taras Banakh

t.o.banakh@gmail.com

1 Jan Kochanowski University in Kielce, Kielce, Poland

2 Ivan Franko National University in Lviv, Lviv, Ukraine
} 
- Borel-measurable if for any Borel set $B \subseteq Y$ the set $\Phi^{-1}[B]$ is Borel in $X$;

- compact-valued if for every $x \in X$ the subspace $\Phi(x)$ of $Y$ is compact and nonempty;

- usco if $\Phi$ is upper semicontinuous and compact-valued.

It is well-known that for any surjective continuous function $f: X \rightarrow Y$ between compact Hausdorff spaces, the inverse multimap $f^{-1}: Y \multimap X$ is usco.

Let $\Phi: X \multimap Y$ be a multimap between topological spaces. A function $f: X \rightarrow Y$ is called a selection of $\Phi$ if $f(x) \in \Phi(x)$ for every $x \in X$. The Axiom of Choice ensures that every multimap $\Phi: X \multimap Y$ with non-empty values has a selection. The problem is to find selections possessing some additional properties like the continuity or measurability.

One of classical results in this direction is the following theorem of Kuratowski and Ryll-Nardzewski [12] (see also [16, §5.2] or [14, 6.12]).

Theorem 1 Let $X, Y$ be Polish spaces. Any Borel-measurable multimap $\Phi: X \multimap Y$ with non-empty values has a Borel-measurable selection.

We recall that a function $f: X \rightarrow Y$ between topological spaces is called Borelmeasurable (resp. $F_{\sigma}$-measurable) if for every open set $U \subseteq Y$ the preimage $f^{-1}[U]$ is Borel (or type $F_{\sigma}$ ) in $X$.

$F_{\sigma}$-Measurable selections of usco multimaps with values in non-metrizable compact spaces were studied by many mathematicians [4-9]. Positive results are known for two classes of compact spaces: fragmentable and linearly ordered.

Let us recall $[3,5.0 .1]$ (see also $[15, \S 6]$ ) that a topological space $K$ is fragmentable if $K$ has a metric $\rho$ such that for every $\varepsilon>0$ each non-empty subset $A \subseteq K$ contains a non-empty relatively open set $U \subseteq A$ of $\rho$-diameter $<\varepsilon$. By [3, 5.1.12], each fragmentable compact Hausdorff space contains a metrizable dense $G_{\delta}$-subspace.

The following selection theorem can be deduced from Theorem 1' and Lemma 6 in [9].

Theorem 2 (Hansell, Jayne, Talagrand) Any usco map $\Phi: X \rightarrow K$ from a perfectly paracompact space $X$ to a fragmentable compact space $Y$ has an $F_{\sigma}$-measurable selection.

A similar selection theorem holds for usco maps into countably cellular GO-spaces. A Hausdorff topological space $X$ is called a generalized ordered space (briefly, a $G O$-space) if $X$ admits a linear order $\leq$ such that the topology of $X$ is generated by a base consisting of open order-convex subsets of $X$. A subset $C$ of a linearly ordered space $X$ is called order-convex if for any points $x \leq y$ in $C$ the order interval $[x, y]:=\{z \in X: x \leq z \leq y\}$ is contained in $X$. We say that the topology of $X$ is generated by the linear order $\leq$ if the topology of $X$ is generated by the subbase $\{(\leftarrow, a),(a, \rightarrow): a \in X\}$ consisting the the order-convex sets $(\leftarrow, a):=\{x \in X:$ $x<a\}$ and $(a, \rightarrow)=\{x \in X: a<x\}$.

A topological space $X$ is countably cellular if every disjoint family of open sets in $X$ is at most countable. It is easy to see that each separable topological space is countably cellular. A topological space is called $F_{\sigma}$-perfect if every open set in $X$ is 
of type $F_{\sigma}$ in $X$ (i.e., can be represented as the countable union of closed sets). For example, every metrizable space is $F_{\sigma}$-perfect.

The following selection theorem will be proved in Sect. 2 .

Theorem 3 Let $Y$ be a $G O$-space and $X$ be an ( $F_{\sigma}$-perfect) topological space. If $X$ or $Y$ is countably cellular, then any usco map $\Phi: X \multimap Y$ has a Borel ( $F_{\sigma}$-measurable $)$ selection.

Theorems 2, 3 suggest the following problem.

Problem 1 Is it true that any usco map $\Phi: M \multimap K$ from a compact metrizable space $M$ to a compact Hausdorff space $K$ has a Borel ( $F_{\sigma}$-measurable) selection?

In this paper we prove that this problem has negative answer under the negation of the Continuum Hypothesis (i.e., under $\omega_{1}<\mathfrak{c}$ ). A suitable counterexample will be constructed using the split square $\ddot{\mathbb{I}}^{2}$, which is the square of the split interval $\ddot{\mathbb{I}}$.

The split interval is the linearly ordered space $\ddot{\mathbb{I}}=[0,1] \times\{0,1\}$ whose topology is generated by the lexicographic order (defined by $\langle x, i\rangle \leq\langle y, j\rangle$ iff either $x<y$ or else $x=y$ and $i \leq j$ ). The split interval plays a fundamental role in the theory of separable Rosenthal compacta [17]. Let us recall that a topological space is called Rosenthal compact if it is homeomorphic to a compact subspace of the space $B_{1}(P)$ of functions of the first Baire class on a Polish space $P$. It is well-known (and easy to see) that the split interval is Rosenthal compact and so is its square. By Theorem 4 of Todorčević [17], each non-metrizable Rosenthal compact space of countable spread contains a topological copy of the split interval. A topological space has countable spread if it contains no uncountable discrete subspaces.

By Theorem 3, any usco map $\Phi: X \multimap \ddot{I}$ from an $F_{\sigma}$-perfect topological space $X$ has an $F_{\sigma}$-measurable selection. In contrast, the split square $\ddot{\mathbb{I}}^{2}$ has dramatically different selections properties. Let $p: \ddot{\mathbb{I}} \rightarrow \mathbb{I}, p:\langle x, i\rangle \mapsto x$, be the natural projection of the split interval onto the unit interval $\mathbb{I}=[0,1]$, and

$$
P: \ddot{\mathbb{I}}^{2} \rightarrow \mathbb{I}^{2}, \quad P:\langle x, y\rangle \mapsto\langle p(x), p(y)\rangle,
$$

be the projection of the split square $\ddot{\mathbb{I}}^{2}$ onto the unit square $\mathbb{I}^{2}$.

Theorem 4 The following conditions are equivalent:

1. the usco multimap $P^{-1}: \mathbb{I}^{2} \rightarrow \ddot{\mathbb{I}}^{2}$ has a Borel-measurable selection;

2. the usco multimap $P^{-1}: \mathbb{I}^{2} \rightarrow \ddot{\mathbb{I}}^{2}$ has an $F_{\sigma}$-measurable selection;

3. $\omega_{1}=\mathfrak{c}$.

The implication $(2) \Rightarrow(1)$ of Theorem 4 is trivial and the implications (1) $\Rightarrow$ (3) $\Rightarrow(2)$ are proved in Lemmas 2 and 8, respectively.

Combining Theorem 4 with Theorem 4 in [17], we obtain the following consistent characterization of metrizable compacta.

Corollary 1 Under $\omega_{1}<\mathfrak{c}$ a Rosenthal compact space $K$ is metrizable if and only if $K$ has countable spread and each usco multimap $\Phi: \mathbb{I}^{2} \rightarrow K^{2}$ has a Borel-measurable selection. 
Proof The "only if" part follows from Theorem 2. To prove the "if" part, assume that a Rosenthal compact $K$ is not metrizable but has countable spread. By Theorem 4 of [17], the space $K$ contains a topological copy of the split interval $\ddot{\mathbb{I}}$. We lose no generality assuming that $\ddot{I} \subseteq K$. By Theorem 4 , under $\omega_{1}<\mathfrak{c}$, the usco multimap $P^{-1}: \mathbb{I}^{2} \multimap \ddot{\mathbb{I}}^{2} \subseteq K^{2}$ does not have Borel-measurable selections.

Now we pose some open problems suggested by Theorem 4 .

Problem 2 Assume $\mathrm{CH}$. Is it true that each usco map $\Phi: X \rightarrow \ddot{\mathbb{I}}^{2}$ from a metrizable (separable) space $X$ has a Borel-measurable selection?

Observe that the map $p: \ddot{\mathbb{I}} \rightarrow \mathbb{I}$ is 2 -to-1 and its square $P: \ddot{\mathbb{I}}^{2} \rightarrow \mathbb{I}^{2}$ is 4-to-1. A function $f: X \rightarrow Y$ is called $n$-to-1 for some $n \in \mathbb{N}$ if $\left|f^{-1}(y)\right| \leq n$ for any $y \in Y$. By Theorem 3 of Todorčević [17], every Rosenthal compact space of countable spread admits a 2-to-1 map onto a metrizable compact space. Let us observe that the splitted square $\ddot{\mathbb{I}}^{2}$ contains a discrete subspace of cardinality continuum and hence has uncountable spread.

Problem 3 Let $n \in\{2,3\}$. Is there an $n$-to-1 map $f: K \rightarrow M$ from a (Rosenthal) compact space $K$ to a metrizable compact space $M$ such that the inverse multimap $f^{-1}: M \multimap K$ has no Borel selections?

Remark 1 hris Heunen asked on Mathoverflow [10] whether for any continuous surjective function $\pi: X \rightarrow Y$ between compact Hausdorff spaces and any continuous map $\gamma: \mathbb{I} \rightarrow Y$ there exists a Borel-measurable function $g: \mathbb{I} \rightarrow X$ such that $\gamma=\pi \circ g$. Theorem 4 provides a consistent counterexample to this problem of Heunen. Indeed, consider the projection $P: \ddot{\mathbb{I}}^{2} \rightarrow \mathbb{I}^{2}$ and take any continuous surjective map $\gamma: \mathbb{I} \rightarrow \mathbb{I}^{2}$ (whose existence was proved by Giuseppe Peano in 1890). By Theorem 2 , the inverse multimap $\gamma^{-1}: \mathbb{I}^{2} \multimap \mathbb{I}$ has a Borel-measurable selection $s: \mathbb{I}^{2} \rightarrow \mathbb{I}$. Assuming that there exists a Borel-measurable function $g: \mathbb{I} \rightarrow \ddot{\mathbb{I}}^{2}$ with $\gamma=P \circ g$, we conclude that $g \circ s: \mathbb{I}^{2} \rightarrow \ddot{\mathbb{I}}^{2}$ is a Borel-measurable selection of the multimap $P^{-1}$, with contradicts Theorem 4 under $\mathrm{CH}$.

\section{Proof of Theorem 3}

Theorem 3 follows from Lemmas 2 and 3, proved in this section.

First we prove one lemma, showing that our definition of a GO-space agrees with the original definition of Lutzer [13]. Probably this lemma is known but we could not find the precise reference in the literature.

Lemma 1 The linear order $\leq$ of any $G O$-space $X$ is a closed subset of the square $X \times X$.

Proof Given two elements $x, y \in X$ with $x \not \leq y$, use the Hausdorff property of $X$ and find two disjoint order-convex neighborhoods $O_{x}, O_{y} \subseteq X$ of the points $x, y$, respectively. We claim that the product $O_{x} \times O_{y}$ is disjoint with the linear order $\leq$. Assuming that this is not true, find elements $x^{\prime} \in O_{x}$ and $y^{\prime} \in O_{y}$ such that $x^{\prime} \leq \overline{y^{\prime}}$. 
Taking into account that the sets $O_{x}, O_{y}$ are disjoint and order-convex, we conclude that $x^{\prime}<y$ and $x<y^{\prime}$. It follows from $x \not \leq y$ that $y<x$. Then $x^{\prime}<y<x<y^{\prime}$ and hence $[y, x] \subseteq O_{x} \cap O_{y}=\emptyset$, which is a desired contradiction. This contradiction shows that the neighborhood $O_{x} \times O_{y}$ of the pair $\langle x, y\rangle$ is disjoint with $\leq$ and hence $\leq$ is a closed subset of $X \times X$.

Lemma 2 Any usco multimap $\Phi: X \multimap Y$ from an $\left(F_{\sigma}\right.$-perfect) topological space $X$ to a countably cellular $G O$-space space $Y$ has a Borel ( $F_{\sigma}$-measurable) selection.

Proof Being a GO-space, $Y$ has a base of the topology consisting of open orderconvex subsets with respect to some linear order $\leq$ on $Y$. By Lemma 1 , the linear order $\leq$ is a closed subset of $Y \times Y$. Then for every $a \in Y$ the order-convex set $(\leftarrow, a]=\{y \in Y: y \leq a\}$ is closed in $Y$, which implies that each non-empty compact subset of $Y$ has the smallest element.

Then for any usco multmap $\Phi: X \multimap Y$ we can define a selection $f: X \rightarrow Y$ of $\Phi$ assigning to each point $x \in X$ the smallest element $f(x)$ of the non-empty compact set $\Phi(x) \subseteq Y$. We claim that this selection is $F_{\sigma}$-measurable.

A subset $U \subseteq Y$ is called upper if for any $u \in U$ the order-convex set $[u, \rightarrow)=$ $\{y \in Y: u \leq y\}$ is contained in $U$.

Claim 1 For any upper open set $U \subseteq Y$ the preimage $f^{-1}[U]$ is open in $X$.

Proof For any $x \in f^{-1}[U]$ we get $\Phi(x) \subseteq[f(x), \rightarrow) \subseteq U$. The upper semicontinuity of $\Phi$ yields a neighborhood $O_{x} \subseteq X$ such that $\Phi\left[O_{x}\right] \subseteq U$. Consequently, $f\left(O_{x}\right) \subseteq$ $\Phi\left[O_{x}\right] \subseteq U$, witnessing that the set $f^{-1}[U]$ is open in $X$.

A subset $L \subseteq Y$ is lower if for every $a \in L$ the order-convex set $(\leftarrow, a]=\{y \in$ $Y: y \leq a\}$ is contained in $L$.

Claim 2 For any closed lower set $L \subseteq Y$ the preimage $f^{-1}[L]$ is closed in $X$.

Proof Observe the the complement $X \backslash L$ is an open upper set in $Y$. By Claim 1, the preimage $f^{-1}[X \backslash L]$ is open in $X$ and hence its complement $X \backslash f^{-1}[X \backslash L]=f^{-1}[L]$ is closed in $X$.

Claim 3 For any lower set $L \subseteq Y$ the preimage $f^{-1}(L)$ is of type $F_{\sigma}$ in $X$.

Proof If $L$ has a largest element $\lambda$, then $L=(\leftarrow, \lambda]$ and $f^{-1}[L]=f^{-1}[(\leftarrow, \lambda]]$ is closed by Claim 2. So, we assume that $L$ does not have a largest element. Then the countable cellularity of $Y$ implies that $L$ has a countable cofinal subset $C \subseteq L$ (which means that for every $x \in L$ there exists $y \in C$ with $x \leq y$ ). By Lemma 2, for every $c \in C$ the preimage $f^{-1}[(\leftarrow, c]]$ is closed in $X$. Since $L=\bigcup_{c \in C}(\leftarrow, c]$, the preimage $f^{-1}[L]=\bigcup_{c \in C} f^{-1}[(\leftarrow, c]]$ is of type $F_{\sigma}$ in $X$.

Claim 4 For any open order-convex subset $U \subseteq Y$ the preimage $f^{-1}[U]$ is a Borel subset of $X$ (of type $F_{\sigma}$ if the space $X$ is $F_{\sigma}$-perfect).

Proof The order-convexity of $U$ implies that $U=\overleftarrow{U} \cap \vec{U}$ where $\overleftarrow{U}=\bigcup_{u \in U}(\leftarrow, u]$ and $\vec{U}=\bigcup_{u \in U}[u, \rightarrow)$. Taking into account that $Y$ has a base of order-convex sets, 
one can show that the upper set $\vec{U}$ is open in $X$. By Claim 1 , the preimage $f^{-1}[\vec{U}]$ is open in $X$ (of type $F_{\sigma}$ if the space $X$ is $F_{\sigma}$-perfect). By Claim 3, the preimage $f^{-1}[\overleftarrow{U}]$ is of type $F_{\sigma}$ in $X$. Then $f^{-1}(U)=f^{-1}[\overleftarrow{U}] \cap f^{-1}[\vec{U}]$ is Borel (of type $F_{\sigma}$ if $X$ is $F_{\sigma}$-perfect).

Claim 5 For every open set $U \subseteq Y$ the preimage $f^{-1}[B]$ is Borel subset of $X$ (of type $F_{\sigma}$ if $X$ is $F_{\sigma}$-perfect).

Proof By the definition of the topology of $Y$, each point $x \in U$ has an open orderconvex neighborhood $O_{x} \subseteq U$. By the Kuratowski-Zorn Lemma, each open orderconvex subset of $U$ is contained in a maximal open order convex subset of $U$. Let $\mathcal{C} \subset \mathcal{B}$ be the family of maximal open order-convex subsets of $U$. Observe that $U=\bigcup \mathcal{C}$ and any distinct sets $C, D \in \mathcal{C}$ are disjoint: otherwise the union $C \cup D$ would be an open order convex subset of $U$ and by the maximality of $C$ and $D, C=C \cup D=D$. Since the space $Y$ is countably cellular, the family $\mathcal{C}$ is at most countable. By Claim 4 , for every $C \in \mathcal{C}$ the preimage $f^{-1}(C)$ is Borel (of type $F_{\sigma}$-set if $X$ is $F_{\sigma}$-perfect) and so is the countable union $f^{-1}[U]=\bigcup_{C \in \mathcal{C}} f^{-1}$.

Claim 5 completes the proof of Lemma 2.

Lemma 3 Every usco multimap $\Phi: X \multimap Y$ from a countably cellular ( $F_{\sigma}$-perfect) topological space $X$ into a $G O$-space $Y$ has a Borel $\left(F_{\sigma}\right.$-measurable) selection.

Proof The Kuratowski-Zorn Lemma implies that the usco map $\Phi$ contains a minimal usco map $\Psi: X \multimap Y$. We claim that the image $\Psi[X] \subseteq Y$ is a countably cellular subspace of $Y$. Assuming the opposite, we can find an uncountable disjoint family $\left(U_{\alpha}\right)_{\alpha \in \omega_{1}}$ of non-empty open subsets in $\Psi[X]$. For every $\alpha \in \omega_{1}$, find $x_{\alpha} \in X$ such that $\Phi\left(x_{\alpha}\right) \cap U_{\alpha} \neq \emptyset$. By Lemma 3.1.2 [3], the minimality of the usco map $\Psi$ implies that $\Psi\left[V_{\alpha}\right] \subseteq U_{\alpha}$ for some non-empty open set $V_{\alpha} \subseteq X$. Taking into account that the family $\left(U_{\alpha}\right)_{\alpha \in \omega_{1}}$ is disjoint, we conclude that the family $\left(V_{\alpha}\right)_{\alpha \in \omega_{1}}$ is disjoint, witnessing that the space $X$ is not countably cellular. But this contradicts our assumption. This contradiction shows that the GO-subspace $\Psi[X]$ of $Y$ is countably cellular. By Lemma 2, the usco map $\Psi: X \rightarrow \Psi[X]$ has a Borel ( $F_{\sigma}$-measurable) selection, which is also a selection of the usco map $\Phi$.

Finally, let us prove one selection property of the split interval, which will be used in the proof of Lemma 8.

Lemma 4 Any selection $s: \ddot{\mathbb{I}} \rightarrow \mathbb{I}$ of the multimap $p^{-1}: \mathbb{I} \rightarrow \ddot{\mathbb{I}}$ is $F_{\sigma}$-measurable.

Proof Given any open subset $U \subseteq \ddot{\mathbb{I}}$, we need to show that $s^{-1}[U]$ is of type $F_{\sigma}$ in $\mathbb{I}$. For every $x \in s^{-1}[U]$, find an open order-convex set $I_{x} \subseteq U$ containing $s(x)$. It is well-known (see e.g. [2, 3.10.C(a)]) that the split interval $\ddot{I}$ is hereditarily Lindelöf. Consequently, there exists a countable set $C \in s^{-1}[U]$ such that $\bigcup_{x \in s^{-1}[U]} I_{x}=$ $\bigcup_{x \in C} I_{x}$ and hence $s^{-1}[U]=\bigcup_{x \in C} s^{-1}\left[I_{x}\right]$. For every $x \in C$ the order-convexity of the interval $I_{x} \subseteq \ddot{\mathbb{I}}$ implies that its preimage $s^{-1}\left[I_{x}\right]$ is a convex subset of $\mathbb{I}$, containing $x$. Since convex subsets of $\mathbb{I}$ are of type $F_{\sigma}$, the countable union $s^{-1}[U]=\bigcup_{x \in C} I_{x}$ is an $F_{\sigma}$-set in $\mathbb{I}$. 


\section{Selection properties of the split square $\ddot{\mathbb{I}}^{2}$ under the negation of $\mathrm{CH}$}

In this section we study the selection properties of the split square $\ddot{\mathbb{I}}^{2}$ under the negation of the Continuum Hypothesis.

By $\langle x, y\rangle$ we denote the ordered pair of points $x, y$. In this way we distinguish ordered pairs from the order intervals $(x, y):=\{z: x<z<y\}$ in linearly ordered spaces.

The split interval $\ddot{\mathbb{I}}=\mathbb{I} \times\{0,1\}$ carries the lexicographic order defined by $\langle x, i\rangle \leq$ $\langle y, j\rangle$ iff either $x<y$ or $(x=y$ and $i \leq j)$. It is well-known that the topology generated by the lexicographic order on $\ddot{\mathbb{I}}$ is compact and Hausdorff, see [2, 3.10.C(b)]. By $p: \ddot{\mathbb{I}} \rightarrow \mathbb{I}, p:\langle x, i\rangle \mapsto x$, we denote the coordinate projection and by $P: \ddot{\mathbb{I}}^{2} \rightarrow \mathbb{I}^{2}$, $P:\langle x, y\rangle \mapsto\langle p(x), p(y)\rangle$ the square of the map $p$.

The following lemma proves the implication $(1) \Rightarrow$ (3) of Theorem 4 .

Lemma 5 If $\omega_{1}<\mathfrak{c}$, then the multimap $P^{-1}: \mathbb{I}^{2} \multimap \ddot{\mathbb{I}}^{2}$ has no Borel selections.

Proof To derive a contradiction, assume that the multimap $P^{-1}$ has a Borel-measurable selection $s: \mathbb{I}^{2} \rightarrow \ddot{\mathbb{I}}^{2}$.

For a real number $x \in \mathbb{I}$ by $x_{0}$ and $x_{1}$ we denote the points $\langle x, 0\rangle$ and $\langle x, 1\rangle$ of the split interval $\ddot{\mathbb{I}}$. Then $\ddot{\mathbb{I}}=\ddot{\mathbb{I}}_{0} \cup \ddot{\mathbb{I}}_{1}$ where $\ddot{\mathbb{I}}_{i}=\left\{x_{i}: x \in \mathbb{I}\right\}$ for $i \in\{0,1\}$.

For any numbers $i, j \in\{0,1\}$ consider the set

$$
Z_{i j}=\left\{z \in \mathbb{I}^{2}: s(z) \in \ddot{\mathbb{I}}_{i} \times \ddot{\mathbb{I}}_{j}\right\}
$$

and observe that $\mathbb{1}^{2}=\bigcup_{i, j=0}^{1} Z_{i j}$.

For a point $a \in \ddot{\mathbb{I}}$, let $\left[0_{0}, a\right)$ and $\left(a, 1_{1}\right]$ be the order intervals in $\ddot{\mathbb{I}}$ with respect to the lexicographic order. Observe that for any $x \in \mathbb{I}$ we have

$$
\begin{array}{ll}
p\left(\left[0_{0}, x_{0}\right)\right)=[0, x), & p\left(\left[0_{0}, x_{1}\right)\right)=[0, x], \\
p\left(\left(x_{0}, 1_{1}\right]\right)=[x, 1], & p\left(\left(x_{1}, 1_{1}\right]\right)=(x, 1] .
\end{array}
$$

For every $a \in(0,2) \subseteq \mathbb{R}$ consider the lines

$$
\mathrm{L}_{a}=\left\{\langle x, y\rangle \in \mathbb{R}^{2}: x+y=a\right\} \text { and } \Gamma^{a}=\left\{\langle x, y\rangle \in \mathbb{R}^{2}: y-x=a\right\}
$$

on the plane.

Claim 6 For every $a \in \mathbb{R}$ the intersection $\mathrm{L}_{a} \cap Z_{00}$ is at most countable.

Proof If for some $a \in \mathbb{R}$ the intersection $\mathrm{L}_{a} \cap Z_{00}$ is uncountable, then we can choose a non-Borel subset $B \subseteq \mathrm{L}_{a} \cap Z_{00}$ of cardinality $|B|=\omega_{1}$. For every point $\langle x, y\rangle \in B \subseteq Z_{00}$, the definition of the set $Z_{00}$ ensures that $s(\langle x, y\rangle)=\left\langle x_{0}, y_{0}\right\rangle$ and hence the set $U_{\langle x, y\rangle}=\left[0_{0}, x_{1}\right) \times\left[0_{0}, y_{1}\right)=\left[0_{0}, x_{0}\right] \times\left[0_{0}, y_{0}\right]$ is an open neighborhood of $s(\langle x, y\rangle)$ in $\ddot{\mathbb{I}}^{2}$. Observe that $\langle x, y\rangle \in s^{-1}\left(U_{\langle x, y\rangle}\right) \subseteq p\left(U_{\langle x, y\rangle}\right)=[0, x] \times[0, y]$ and hence $\mathrm{L}_{a} \cap s^{-1}\left(U_{\langle x, y\rangle}\right)=\{\langle x, y\rangle\}$. Then for the open set $U=\bigcup_{\langle x, y\rangle \in B} U_{\langle x, y\rangle}$ the preimage $s^{-1}[U]$ is not Borel in $\mathbb{I}^{2}$ because the intersection $s^{-1}[U] \cap \mathrm{L}_{a}=B$ is not Borel. But this contradicts the Borel measurability of $s$. 
By analogy we can prove the following claims.

Claim 7 For every $a \in \mathbb{R}$ the intersection $\mathrm{L}_{a} \cap Z_{11}$ is at most countable.

Claim 8 For every $b \in \mathbb{R}$ the intersection $\Gamma^{b} \cap\left(Z_{01} \cup Z_{10}\right)$ is at most countable.

Now fix any subset set $\Omega \subseteq\left[\frac{1}{2}, \frac{3}{2}\right]$ of cardinality $|\Omega|=\omega_{1}$. By Claims 6,7 , for every $a \in \Omega$ the intersection $\mathrm{L}_{a} \cap\left(Z_{00} \cup Z_{11}\right)$ is at most countable. Consequently the union

$$
U=\bigcup_{a \in \Omega} \mathrm{L}_{a} \cap\left(Z_{00} \cup Z_{11}\right)
$$

has cardinality $|U| \leq \omega_{1}$. Since $|U| \leq \omega_{1}<\mathfrak{c}$, there exists a real number $b \in\left[\frac{1}{2}, \frac{3}{2}\right]$ such that the line $\Gamma^{b}$ does not intersect the set $U$. Since $\{b\} \cup \Omega \subseteq\left[\frac{1}{2}, \frac{3}{2}\right]$ for every $a \in \Omega$ the intersection $\Gamma^{b} \cap \mathrm{L}_{a} \cap \mathbb{I}^{2}$ is not empty. Then the set $X=\bigcup_{a \in \Omega} \mathrm{L}_{a} \cap \Gamma^{b} \subset \mathbb{I}^{2}$ is uncountable and $X \subseteq \Gamma^{b} \backslash U \subseteq \Gamma^{b} \cap\left(Z_{01} \cup Z_{10}\right)$. But this contradicts Claim 8 .

\section{Selection properties of the split square $\ddot{\mathbb{I}}^{2}$ under the Continuum Hypothesis}

In this section we shall prove that under the continuum hypothesis the usco multimap $P^{-1}: \mathbb{I}^{2} \multimap \ddot{\mathbb{I}}^{2}$ has an $F_{\sigma}$-measurable selection.

First we introduce some terminology related to monotone functions.

A subset $f \subseteq \mathbb{I}^{2}$ is called

- a function if for any $\langle x, y\rangle,\left\langle x^{\prime}, y^{\prime}\right\rangle \in f$ the equality $x=x^{\prime}$ implies $y=y^{\prime}$;

- strictly increasing if for any $\langle x, y\rangle,\left\langle x^{\prime}, y^{\prime}\right\rangle \in f$ the strict inequality $x<x^{\prime}$ implies $y<y^{\prime}$

- strictly decreasing if for any $\langle x, y\rangle,\left\langle x^{\prime}, y^{\prime}\right\rangle \in f$ the inequality $x<x^{\prime}$ implies $y>y^{\prime}$;

- strictly monotone if $f$ is strictly increasing or strictly decreasing.

Lemma 6 Each strictly increasing function $f \subset \mathbb{I}^{2}$ is a subset of a Borel strictly increasing function $\bar{f} \subset \mathbb{I}^{2}$.

Proof It follows that the strictly increasing function $f$ is a strictly increasing bijective function between the sets $\operatorname{pr}_{1}[f]=\{x \in \mathbb{I}: \exists y \in \mathbb{I}\langle x, y\rangle \in f\}$ and $\operatorname{pr}_{2}[f]=$ $\{y \in \mathbb{I}: \exists x \in \mathbb{I} \quad\langle x, y\rangle \in f\}$. It is well-known that monotone functions of one real variable have at most countably many discontinuity points. Consequently, the sets of discontinuity points of the strictly monotone functions $f$ and $f^{-1}$ are at most countable. This allows us to find a countable set $D_{f} \subseteq f$ such that the set $f \backslash D_{f}$ coincides with the graph of some increasing homeomorphism between subsets of $\mathbb{I}$. Replacing $D_{f}$ by a larger countable set, we can assume that $D_{f}=f \cap\left(\operatorname{pr}_{1}\left[D_{f}\right] \times\right.$ $\left.\operatorname{pr}_{2}\left[D_{f}\right]\right)$, where $\operatorname{pr}_{1}, \operatorname{pr}_{2}: \mathbb{I}^{2} \rightarrow \mathbb{I}$ are coordinate projections. By the Lavrentiev Theorem [11, 3.9], the homeomorphism $f \backslash D_{f}$ extends to a (strictly increasing) homeomorphism $h \subseteq \mathbb{I}^{2}$ between $G_{\delta}$-subsets of $\mathbb{I}^{2}$ such that $f \backslash D_{f}$ is dense in $h$. It is easy to check that the Borel subset $\bar{f}=\left(h \backslash\left(\operatorname{pr}_{1}\left[D_{f}\right] \times \operatorname{pr}_{2}\left[D_{f}\right]\right) \cup D_{f}\right.$ is a strictly increasing function extending $f$. 
By analogy we can prove

Lemma 7 Each strictly decreasing function $f \subset \mathbb{I}^{2}$ is a subset of a Borel strictly decreasing function $\bar{f} \subset \mathbb{I}^{2}$.

Now we are ready to prove the main result of this section.

Lemma 8 Under $\omega_{1}=\mathfrak{c}$ the multifunction $P^{-1}: \mathbb{I}^{2} \multimap \ddot{\mathbb{I}}^{2}$ has an $F_{\sigma}$-measurable selection.

Proof Let $\mathcal{M}$ be the set of infinite strictly monotone Borel functions $f \subset \mathbb{I}^{2}$. Since $\omega_{1}=\mathfrak{c}$, the set $\mathcal{M}$ can be written as $\mathcal{M}=\left\{f_{\alpha}\right\}_{\alpha<\omega_{1}}$. It is clear $\bigcup_{\alpha<\omega_{1}} f_{\alpha}=\mathbb{I}^{2}$. So, for any point $z \in \mathbb{I}^{2}$ we can find the smallest ordinal $\alpha_{z}<\omega_{1}$ such that $z \in f_{\alpha_{z}}$. Consider the sets

$$
\begin{aligned}
& \mathrm{L}:=\left\{z \in \mathbb{I}^{2}: f_{\alpha_{z}} \text { is strictly increasing }\right\} \text { and } \\
& \Gamma:=\left\{z \in \mathbb{I}^{2}: f_{\alpha_{z}} \text { is strictly decresing }\right\}=\mathbb{I}^{2} \backslash \mathrm{L} .
\end{aligned}
$$

Define a selection $s: \mathbb{I}^{2} \rightarrow \ddot{\mathbb{I}}^{2}$ of the multimap $P^{-1}: \mathbb{I}^{2} \multimap \ddot{\mathbb{I}}^{2}$ letting

$$
s(\langle x, y\rangle)= \begin{cases}\left\langle x_{1}, y_{1}\right\rangle & \text { if }\langle x, y\rangle \in \mathrm{L} \\ \left\langle x_{1}, y_{0}\right\rangle & \text { if }\langle x, y\rangle \in \Gamma\end{cases}
$$

for $\langle x, y\rangle \in \mathbb{I}^{2}$.

We claim that the function $s: \mathbb{I}^{2} \rightarrow \ddot{\mathbb{I}}^{2}$ is $F_{\sigma}$-measurable. Given any open set $U \subseteq \ddot{\mathbb{I}}^{2}$, we should prove that its preimage $s^{-1}[U]$ of type $F_{\sigma}$ in $\mathbb{I}^{2}$. Consider the open subset $V:=U \cap\left(0_{1}, 1_{0}\right)^{2} \subseteq \ddot{\mathbb{I}}^{2}$ of $U$. Using Lemma 4, it can be shown that the set $s^{-1}[U \backslash V] \subseteq \mathbb{I}^{2} \backslash(0,1)^{2}$ is of type $F_{\sigma}$ in $\mathbb{I}^{2}$. Therefore, it remains to show that the preimage $s^{-1}[V]$ is of type $F_{\sigma}$ in $\mathbb{I}^{2}$.

Let $\mathbb{Q}:=\left\{\frac{n}{m}: n, m \in \mathbb{N}, n<m\right\}$ be the set of rational numbers in the interval $(0,1)$.

Consider the subsets $\mathrm{L}_{V}:=\mathrm{L} \cap s^{-1}(V)$ and $\Gamma_{V}:=\Gamma \cap s^{-1}(V)$. For every $\langle x, y\rangle \in$ $\mathrm{L}_{V}$ we have $s(\langle x, y\rangle)=\left\langle x_{1}, y_{1}\right\rangle \in V$ and by the definition of the topology of the split interval, we can find rational numbers $a(x, y), b(x, y) \in \mathbb{Q}$ such that $x<a(x, y)$, $y<b(x, y)$ and $s(\langle x, y\rangle)=\left\langle x_{1}, y_{1}\right\rangle \in\left[x_{1}, a(x, y)_{0}\right) \times\left[y_{1}, b(x, y)_{0}\right) \subseteq V$. Then

$$
[x, a(x, y)) \times[y, b(x, y))=s^{-1}\left[\left[x_{1}, a(x, y)_{0}\right) \times\left[y_{1}, b(x, y)_{0}\right)\right] \subseteq s^{-1}[V] .
$$

On the other hand, for every $\langle x, y\rangle \in \Gamma_{V}$ there are rational numbers $a(x, y), b(x, y)$ $\in \mathbb{Q}$ such that $x<a(x, y), b(x, y)<y$ and $s(\langle x, y\rangle)=\left\langle x_{1}, y_{0}\right\rangle=\left[x_{1}, a(x, y)_{0}\right) \times$ $\left(b(x, y)_{1}, y_{0}\right] \subseteq V$. In this case

$$
[x, a(x, y)) \times(b(x, y), y]=s^{-1}\left[\left[x_{1}, a(x, y)_{0}\right) \times\left(b(x, y)_{1}, y_{0}\right]\right) \subseteq s^{-1}[V] .
$$

It follows that

$$
s^{-1}[V]=\left(\bigcup_{\langle x, y\rangle \in \mathrm{L}_{V}}[x, a(x, y)) \times[y, b(x, y))\right)
$$




$$
\cup\left(\bigcup_{\langle x, y\rangle \in \Gamma_{V}}[x, a(x, y)) \times(b(x, y), y]\right) .
$$

This equality and the following claim imply that the set $s^{-1}[V]$ is of type $F_{\sigma}$ in $\mathbb{I}^{2}$.

Claim 9 There are countable subsets $\mathrm{L}^{\prime} \subseteq \mathrm{L}_{V}$ and $\Gamma^{\prime} \subseteq \Gamma_{V}$ such that

$$
\bigcup_{\langle x, y\rangle \in \mathrm{L}_{V}}[x, a(x, y)) \times[y, b(x, y))=\bigcup_{\langle x, y\rangle \in \mathrm{L}^{\prime}}[x, a(x, y)) \times[y, b(x, y))
$$

and

$$
\bigcup_{\langle x, y\rangle \in \Gamma_{V}}[x, a(x, y)) \times(b(x, y), y]=\bigcup_{\langle x, y\rangle \in \Gamma^{\prime}}[x, a(x, y)) \times(b(x, y), y] .
$$

We shall show how to find the countable set $\mathrm{L}^{\prime} \subseteq \mathrm{L}_{V}$. The countable set $\Gamma^{\prime} \subseteq \Gamma_{V}$ can be found by analogy.

For rational numbers $r, q \in \mathbb{Q}$, consider the set

$$
\mathrm{L}_{r, q}=\left\{\langle x, y\rangle \in \mathrm{L}_{V}: a(x, y)=r, b(x, y)=q\right\}
$$

and observe that $\mathrm{L}_{V}=\bigcup_{r, q \in \mathbb{Q}} \mathrm{L}_{p, q}$.

Claim 10 For any rational numbers $r, q \in \mathbb{Q}$ there exists a countable subset $\mathrm{L}_{r, q}^{\prime} \subseteq$ $\mathrm{L}_{r, q}$ such that

$$
\bigcup_{\langle x, y\rangle \in \mathrm{L}_{r, q}^{\prime}}[x, r) \times[y, q)=\bigcup_{\langle x, y\rangle \in \mathrm{L}_{r, q}}[x, r) \times[y, q) .
$$

Proof For every rational numbers $r^{\prime} \leq r$ and $q^{\prime} \leq q$, consider the numbers $\underline{y}\left(r^{\prime}\right):=\inf \left\{y:\langle x, y\rangle \in \mathrm{L}_{r, q}, x<r^{\prime}\right\}$ and $\underline{x}\left(q^{\prime}\right):=\inf \left\{x:\langle x, y\rangle \in \mathrm{L}_{r, q}, y<q^{\prime}\right\}$.

Choose countable subsets $\mathrm{L}_{r, q}^{r^{\prime}, 0}, L_{r, q}^{0, q^{\prime}} \subseteq \mathrm{L}_{r, q}$ such that $\underline{y}\left(r^{\prime}\right)=\inf \left\{y:\langle x, y\rangle \in \mathrm{L}_{r, q}^{r^{\prime}, 0}, x<r^{\prime}\right\}$ and $\underline{x}\left(q^{\prime}\right)=\inf \left\{x:\langle x, y\rangle \in \mathrm{L}_{r, q}^{0, q^{\prime}}, y<q^{\prime}\right\}$ and moreover, $\underline{y}\left(r^{\prime}\right)=\min \left\{y:\langle x, y\rangle \in \mathrm{L}_{r, q}^{r^{\prime}, 0}, x<r^{\prime}\right\}$ if $\underline{y}\left(r^{\prime}\right)=\min \left\{y:\langle x, y\rangle \in \mathrm{L}_{r, q}, x<r^{\prime}\right\}$. and $\underline{x}\left(q^{\prime}\right)=\min \left\{x:\langle x, y\rangle \in \mathrm{L}_{r, q}^{0, q^{\prime}}, y<q^{\prime}\right\}$ if $\underline{x}\left(q^{\prime}\right)=\min \left\{x:\langle x, y\rangle \in \mathrm{L}_{r, q}, y<q^{\prime}\right\}$. 
Consider the countable subset

$$
\mathrm{L}_{r, q}^{\prime \prime}:=\bigcup\left\{\mathrm{L}_{r, q}^{r^{\prime}, 0} \cup \mathrm{L}_{r, 1}^{0, q^{\prime}}: r^{\prime}, q^{\prime} \in \mathbb{Q}, r^{\prime}<r, q^{\prime}<q\right\}
$$

of $\mathrm{L}_{r, q}$.

$$
\text { Claim } 11 \bigcup_{\langle x, y\rangle \in L_{r, q}}([x, r) \times[y, q)) \backslash\{\langle x, y\rangle\} \subseteq \bigcup_{\langle x, y\rangle \in \mathrm{L}_{r, q}^{\prime \prime}}[x, r) \times[y, q) .
$$

Proof Fix any pairs $\langle x, y\rangle \in \mathrm{L}_{r, q}$ and $\left\langle x^{\prime}, y^{\prime}\right\rangle \in([x, r) \times[y, q)) \backslash\{\langle x, y\rangle\}$. Three cases are possible:

1. $x<x^{\prime}<r$ and $y<y^{\prime}<q$;

2. $x=x^{\prime}$ and $y<y^{\prime}<q$;

3. $x<x^{\prime}<r$ and $y=y^{\prime}$.

In the first case there exist rational numbers $r^{\prime}, q^{\prime}$ such that $x<r^{\prime}<x^{\prime}<r$ and $y<q^{\prime}<y^{\prime}<q$. The definition of $\underline{x}\left(q^{\prime}\right)$ ensures that $\underline{x}\left(q^{\prime}\right) \leq x<x^{\prime}$. By the choice of the family $\mathrm{L}_{r, q}^{0, q^{\prime}}$, there exists $\left\langle x^{\prime \prime}, y^{\prime \prime}\right\rangle \in \mathrm{L}_{r, q}^{0, q^{\prime}} \subset \mathrm{L}_{r, q}^{\prime \prime}$ such that $x^{\prime \prime}<x^{\prime}<r$ and $y^{\prime \prime}<q^{\prime}<y^{\prime}<q$. Then $\left\langle x^{\prime}, y^{\prime}\right\rangle \in\left[x^{\prime \prime}, r\right) \times\left[y^{\prime \prime}, q\right)$.

Next, assume that $x=x^{\prime}$ and $y<y^{\prime}<q$. In this case we can choose a rational number $q^{\prime}$ such that $y<q^{\prime}<y^{\prime}$. It follows that $\underline{x}\left(q^{\prime}\right) \leq x=x^{\prime}$. If $\underline{x}\left(q^{\prime}\right)<x^{\prime}$, then by the definition of the family $\mathrm{L}_{r, q}^{0, q^{\prime}}$, there exists $\left\langle x^{\prime \prime}, y^{\prime \prime}\right\rangle \in \mathrm{L}_{r, q}^{0, q^{\prime}} \subseteq \mathrm{L}_{r, q}$ such that $x^{\prime \prime}<x^{\prime}<r$ and $y^{\prime \prime}<q^{\prime}<y^{\prime}<q$. Then $\left\langle x^{\prime}, y^{\prime}\right\rangle \in\left[x^{\prime \prime}, r\right) \times\left[y^{\prime \prime}, q\right)$.

So, we assume that $\underline{x}\left(q^{\prime}\right)=x^{\prime}=x$ and hence $\underline{x}\left(q^{\prime}\right)=x=\min \left\{x^{\prime \prime}:\left\langle x^{\prime \prime}, y^{\prime \prime}\right\rangle \in\right.$ $\left.\mathrm{L}_{p, q}: y^{\prime \prime}<q^{\prime}\right\}$. In this case $x^{\prime}=\underline{x}\left(q^{\prime}\right)=x^{\prime \prime}$ for some $\left\langle x^{\prime \prime}, y^{\prime \prime}\right\rangle \in \mathrm{L}_{r, q}^{0, q^{\prime}} \subset \mathrm{L}_{r, q}^{\prime \prime}$ with $y^{\prime \prime}<q^{\prime}<y^{\prime}<q$. Then $\left\langle x^{\prime}, y^{\prime}\right\rangle \in\left[x^{\prime \prime}, r\right) \times\left[y^{\prime \prime}, q\right)$.

By analogy, in the third case $\left(x<x^{\prime}<r\right.$ and $\left.y=y^{\prime \prime}\right)$ we can find a pair $\left\langle x^{\prime \prime}, y^{\prime \prime}\right\rangle \in L_{r, q}^{\prime \prime}$ such that $\left\langle x^{\prime}, y^{\prime}\right\rangle \in\left[x^{\prime \prime}, r\right) \times\left[y^{\prime \prime}, q\right)$.

Claim 11 implies that the set

$$
D_{r, q}=\left(\bigcup_{\langle x, y\rangle \in \mathrm{L}_{r, q}}[x, r) \times[y, q)\right) \backslash\left(\bigcup_{\langle x, y\rangle \in \mathrm{L}_{r, q}^{\prime \prime}}[x, r) \times[y, q)\right)
$$

is contained in $\mathrm{L}_{r, q}$.

Claim 12 The set $D_{r, q}$ is a strictly decreasing function.

Proof First we show that $D_{r, q}$ is a function. Assuming that $D_{r, q}$ is not a function, we can find two pairs $\langle x, y\rangle,\left\langle x, y^{\prime}\right\rangle \in D_{r, q}$ with $y<y^{\prime}$. Applying Claim 11, we conclude that

$$
\left\langle x, y^{\prime}\right\rangle \in([x, r) \times[y, q)) \backslash\{\langle x, y\rangle\} \subseteq \bigcup_{\left\langle x^{\prime \prime}, y^{\prime \prime}\right\rangle \in L_{r, q}^{\prime \prime}}\left[x^{\prime \prime}, r\right) \times\left[y^{\prime \prime}, q\right)
$$

and hence $\left\langle x, y^{\prime}\right\rangle \notin D_{r, q}$, which contradicts the choice of the pair $\left\langle x, y^{\prime}\right\rangle$. This contradiction shows that $D_{r, q}$ is a function. 
Assuming that $D_{r, q}$ is not strictly decreasing, we can find pairs $\langle x, y\rangle,\left\langle x^{\prime}, y^{\prime}\right\rangle \in$ $D_{r, q}$ such that $x<x^{\prime}$ and $y \leq y^{\prime}$. Applying Claim 11, we conclude that

$$
\left\langle x^{\prime}, y^{\prime}\right\rangle \in([x, r) \times[y, q)) \backslash\{\langle x, y\rangle\} \subseteq \bigcup_{\left\langle x^{\prime \prime}, y^{\prime \prime}\right\rangle \in L_{r, q}^{\prime \prime}}\left[x^{\prime \prime}, r\right) \times\left[y^{\prime \prime}, q\right)
$$

and hence $\left\langle x^{\prime}, y^{\prime}\right\rangle \notin D_{r, q}$, which contradicts the choice of the pair $\left\langle x, y^{\prime}\right\rangle$. This contradiction shows that $D_{r, q}$ is strictly decreasing.

Claim 13 The set $D_{r, q}$ is at most countable.

Proof To derive a contradiction, assume that $D_{r, q}$ is uncountable. By Lemma 7, the strictly decreasing function $D_{r, q}$ is contained in some Borel strictly decreasing function, which is equal to $f_{\alpha}$ for some ordinal $\alpha<\omega_{1}$. Since the intersection of a strictly increasing function and a strictly decreasing function contains at most one point, the set

$$
D_{r, q}^{\prime}=\bigcup\left\{D_{r, q} \cap f_{\beta}: \beta \leq \alpha, f_{\beta} \text { is strictly increasing }\right\}
$$

is at most countable. We claim that $D_{r, q}=D_{r, q}^{\prime}$. To derive a contradiction, assume that $D_{r, q} \backslash D_{r, q}^{\prime}$ contains some pair $z=\langle x, y\rangle$. It follows from $z \in D_{r, q} \subseteq f_{\alpha}$ that $\alpha_{z} \leq \alpha$. Since $z \notin D_{r, q}^{\prime}$, the strictly monotone function $f_{\alpha_{z}} \ni z$ is not strictly increasing and hence $f_{\alpha_{z}}$ is strictly decreasing. Then the definition of the set $\mathrm{L}$ guarantees that $z \notin \mathrm{L}$, which contradicts the inclusion $z \in D_{r, q} \subseteq \mathrm{L}_{r, q} \subseteq \mathrm{L}$.

Now consider the countable subset $\mathrm{L}_{r, q}^{\prime}:=\mathrm{L}_{r, q}^{\prime \prime} \cup D_{r, q}$ of $\mathrm{L}_{r, q}$ and observe that

$$
\bigcup_{\langle x, y\rangle \in L_{r, q}}([x, r) \times[y, q)) \subseteq \bigcup_{\langle x, y\rangle \in \mathrm{L}_{r, q}^{\prime \prime}}[x, r) \times[y, q) .
$$

This completes the proof of Claim 10.

Claim 14 There exists a countable subset $\mathrm{L}^{\prime} \subseteq \mathrm{L}_{V}$ such that

$$
\bigcup_{\langle x, y\rangle \in \mathrm{L}^{\prime}}\left([x, a(x, y)) \times[y, b(x, y))=\bigcup_{\langle x, y\rangle \in \mathrm{L}_{V}}([x, a(x, y)) \times[y, b(x, y)) .\right.
$$

Proof By Claim 10, for any rational numbers $r, q \in \mathbb{Q}$ there exists a countable subset $\mathrm{L}_{r, q}^{\prime} \subseteq \mathrm{L}_{r, q}$ such that

$$
\begin{aligned}
\bigcup_{\langle x, y\rangle \in \mathrm{L}_{r, q}^{\prime}}([x, a(x, y)) \times[y, b(a, y))) & =\bigcup_{\langle x, y\rangle \in \mathrm{L}_{r, q}^{\prime}}([x, r) \times[y, q))= \\
= & \bigcup_{\langle x, y\rangle \in \mathrm{L}_{r, q}}([x, r) \times[y, q))=\bigcup_{\langle x, y\rangle \in \mathrm{L}_{r, q}}([x, a(x, y)) \times[y, b(x, y))) .
\end{aligned}
$$

Since $\mathrm{L}_{V}=\bigcup_{r, q \in \mathbb{Q}} \mathrm{L}_{r, q}$, the countable set $\mathrm{L}^{\prime}:=\bigcup_{r, q \in \mathbb{Q}} \mathrm{L}_{r, q}^{\prime}$ has the required property. 


\section{By analogy with Claim 14 we can prove}

\section{Claim 15 There exists a countable subset $\Gamma^{\prime} \subseteq \Gamma_{V}$ such that}

$$
\bigcup_{\langle x, y\rangle \in \Gamma^{\prime}}([x, a(x, y)) \times(b(x, y), y])=\bigcup_{\langle x, y\rangle \in \Gamma_{V}}([x, a(x, y)) \times(b(x, y), y]) .
$$

\section{Claims 14 and 15 complete the proof of Claim 9 and the proof of Lemma 8.}

Acknowledgements The author expresses his sincere thanks to Dušan Repovš for vaulable information of measurable selectors, and to Yves Cornulier for helpful comments to the question, posed by the author on Mathoverflow [1], and suggesting the idea of the proof of Lemma 5.

Open Access This article is licensed under a Creative Commons Attribution 4.0 International License, which permits use, sharing, adaptation, distribution and reproduction in any medium or format, as long as you give appropriate credit to the original author(s) and the source, provide a link to the Creative Commons licence, and indicate if changes were made. The images or other third party material in this article are included in the article's Creative Commons licence, unless indicated otherwise in a credit line to the material. If material is not included in the article's Creative Commons licence and your intended use is not permitted by statutory regulation or exceeds the permitted use, you will need to obtain permission directly from the copyright holder. To view a copy of this licence, visit http://creativecommons.org/licenses/by/4.0/.

\section{References}

1. Banakh, T.: A Non-Borel Union of Unit Half-Open Squares. https://mathoverflow.net/q/330488

2. Engelking, R.: General topology. Heldermann Verlag, Berlin (1989)

3. Fabian, M.: Gâteaux Differentiability of Convex Functions and Topology. Weak Asplund Spaces. A Wiley-Interscience Publication. Wiley, New York (1997)

4. Jayne, J.E., Rogers, C.A.: Sélections boréliennes de multi-applications semi-continues supérieurement. C. R. Acad. Sci. Paris Ser. I Math. 299(5), 125-128 (1984)

5. Jayne, J.E., Rogers, C.A.: Borel selectors for upper semicontinuous multivalued functions. J. Funct. Anal. 56(3), 279-299 (1984)

6. Jayne, J.E., Rogers, C.A.: Borel selectors for upper semicontinuous multivalued functions. Mathematika 32(2), 324-337 (1985)

7. Jayne, J.E., Orihuela, J., Pallarés, A.J., Vera, G.: $\sigma$-fragmentability of multivalued maps and selection theorems. J. Funct. Anal. 117(2), 243-273 (1993)

8. Hansell, R.W.: First class selectors for upper semicontinuous multifunctions. J. Funct. Anal. 75(2), 382-395 (1987)

9. Hansell, R.W., Jayne, J.E., Talagrand, M.: First class selectors for weakly upper semicontinuous multivalued maps in Banach spaces. J. Reine Angew. Math. 361, 201-220 (1985)

10. Heunen, C.: Do Continuous Maps Factor Through Continuous Surjections via Borel Maps? https:// mathoverflow.net/q/329935

11. Kechris, A.: Classical Descriptive Set Theory. Springer, New York (1995)

12. Kuratowski, K., Ryll-Nardzewski, C.: A general theorem on selectors. Bull. Polish Acad. des Sci. 13, 397-403 (1965)

13. Lutzer, D.J.: On generalized ordered Spaces. Dissertationes Math. Rozprawy Mat. 89, 1-32 (1971)

14. Repovš, D., Semenov, P.: Continuous Selections of Multivalued Mappings. Kluwer, Dordrecht (1998)

15. Shakhmatov, D.: Compact spaces and their generalizations. Recent progress in general topology (Prague, 1992). North-Holland, Amsterdam (1991) pp 571-640

16. Srivastava, S.M.: A Course on Borel Sets. Springer, New York (1998)

17. Todorčević, S.: Compact subsets of the first Baire class. J. Am. Math. Soc. 12(4), 1179-1212 (1999)

Publisher's Note Springer Nature remains neutral with regard to jurisdictional claims in published maps and institutional affiliations. 